\title{
Incidence of Missed Diagnosed COVID-19 Pneumonia on Patients Who Admitted Urology Outpatient Clinic
}

\author{
Mehmet Serkan Ozkent ${ }^{1}$, Burak Ylmaz ${ }^{1}$, Mustafa Hamarat ${ }^{2}$, and Esma Eroglu ${ }^{3}$ \\ ${ }^{1}$ Konya City Hospital \\ ${ }^{2}$ University of Health Sciences \\ ${ }^{3}$ Konya Meram State Hospital
}

April 27, 2021

\begin{abstract}
Purpose: The novel coronavirus disease 2019 (COVID-19) has spread all over the world. The diagnosis of COVID-19 in asymptomatic patients and patients with non-respiratory symptoms remains a big concern. In this study, we aimed to evaluate the incidence of missed diagnosed COVID-19 pneumonia on abdominal computed tomography (CT) performed in patients admitted to our urology outpatient clinic. Methods: We reviewed the files of patients who were admitted to the urology outpatient clinic from 1 April to 1 November retrospectively. We included the patients who performed abdominal CT at the urology outpatient clinic for any reason and recorded demographic data and abdominal CT findings. We excluded patients with pulmonary symptoms and previously diagnosed with COVID-19. Also, patients without abdominal CT were excluded. We evaluated the rates of missed diagnosed COVID-19 pneumonia detection on the lung base images of abdominal CT. Results: 1024 patients were included in this study. We observed that $99(9.7 \%)$ of these patients had findings related to COVID-19 pneumonia on the lung base images of abdominal CT. Although 885 (86.4\%) patients had no pathological pulmonary findings, $40(3.9 \%)$ patients had other pathological pulmonary findings. Conclusion: COVID-19 disease has become a pandemic all over the world. All healthcare professionals, including urologists, play an active role in the diagnosis and treatment of this disease. So, it should be kept in mind that COVID-19 pneumonia should be evaluated in patients admitted to the urology outpatient clinic with renal colic or abdominal pain.
\end{abstract}

\section{Incidence of Missed Diagnosed COVID-19 Pneumonia on Patients Who Admitted Urology Outpatient Clinic}

Mehmet Serkan Ozkent ${ }^{1}$, Burak Yılmaz ${ }^{1}$, Mustafa Bilal Hamarat ${ }^{1}$, Esma Eroğlu ${ }^{2}$

1, Department of Urology, Konya City Hospital, Health Sciences University, Konya, Turkey.

2, Department of Infectious Diseases and Clinical Microbiology, Meram State Hospital, Konya, Turkey.

Running Title: Incidence of Missed Diagnosed COVID-19 Pneumonia

Keywords: The novel coronavirus disease 2019, COVID-19, computed tomography, urology

Corresponding author: Mehmet Serkan Ozkent, MD.

Department of Urology, Konya City Hospital, Health Sciences University, Konya, Turkey.

ORCID ID: https://orcid.org/0000-0002-6613-0671

E-Mail : msozkent@gmail.com

Phone: +90 (505) 9364155 
Fax: $+90(332) 2236044$

Address: Akabe, Karatay/Konya; Department of Urology, Konya City Hospital, Health Sciences University, 42020, Konya, Turkey

Burak Yilmaz, MD.

E-mail: drburakyilmaz@hotmail.com

Phone Number: +90 (542) 6038367

ORCID ID: https://orcid.org/0000-0002-4242-9134

Address: Department of Urology, Konya City Hospital, Health Sciences University, Konya, Turkey

Mustafa Bilal Hamarat, Assistant Prof.

E-mail: bilalhamarat@gmail.com

Phone Number: +90 (533) 6593586

ORCID ID: https://orcid.org/0000-0002-3987-7016

Address: Department of Urology, Konya City Hospital, Health Sciences University, Konya, Turkey

Esma Eroğlu, MD.

E-mail: esmagulesen@hotmail.com

Phone Number: +90 (554) 9625477

ORCID ID: https://orcid.org/0000-0003-1469-9978

Address: Department of Infectious Diseases and Clinical Microbiology, Meram State Hospital, Konya, Turkey

Author Disclosure Statement

Conflicts of interests

The authors declare that they have no competing interests exist.

Ethics declarations

The institutional human research ethics committee approved the protocol 20/483 (Health Science University, Hamidiye Scientific Research Ethics Committee). The analysis and data collection were performed following the Declaration of Helsinki after written informed consent was obtained from all patients.

Funding

The authors declare that they have no financial competing interests exist.

Availability of data and material

Data are available on request.

Code availability

Not applicable

Acknowledgments

None

Abstract 
Purpose: The novel coronavirus disease 2019 (COVID-19) has spread all over the world. The diagnosis of COVID-19 in asymptomatic patients and patients with non-respiratory symptoms remains a big concern. In this study, we aimed to evaluate the incidence of missed diagnosed COVID-19 pneumonia on abdominal computed tomography (CT) performed in patients admitted to our urology outpatient clinic.

Methods: We reviewed the files of patients who were admitted to the urology outpatient clinic from 1 April to 1 November retrospectively. We included the patients who performed abdominal CT at the urology outpatient clinic for any reason and recorded demographic data and abdominal CT findings. We excluded patients with pulmonary symptoms and previously diagnosed with COVID-19. Also, patients without abdominal CT were excluded. We evaluated the rates of missed diagnosed COVID-19 pneumonia detection on the lung base images of abdominal CT.

Results: 1024 patients were included in this study. We observed that $99(9.7 \%)$ of these patients had findings related to COVID-19 pneumonia on the lung base images of abdominal CT. Although 885 (86.4\%) patients had no pathological pulmonary findings, $40(3.9 \%)$ patients had other pathological pulmonary findings.

Conclusion: COVID-19 disease has become a pandemic all over the world. All healthcare professionals, including urologists, play an active role in the diagnosis and treatment of this disease. So, it should be kept in mind that COVID-19 pneumonia should be evaluated in patients admitted to the urology outpatient clinic with renal colic or abdominal pain.

Keywords: The novel coronavirus disease 2019, COVID-19, computed tomography, urology.

\section{What's already known about this topic?}

- The presence of these nonspecific symptoms may cause delaying the diagnosis of the disease.

- The early diagnosis plays an important role to prevent the spread of the disease and avoids the rapidly deteriorating patients with COVID-19

- In addition, the diagnosis of COVID-19 in asymptomatic patients and patients with non-respiratory symptoms remains a big concern.

\section{What does this article add?}

All of $9.7 \%$ these patients had findings related to COVID-19 pneumonia on the lung base images of abdominal CT.

It should be kept in mind that COVID-19 pneumonia should be evaluated in patients admitted to the urology outpatient clinic with renal colic or abdominal pain.

\section{Main Text}

\section{Introduction}

The novel coronavirus disease 2019 (COVID-19) has spread all over the world(1). The pathogen has been named novel severe acute respiratory syndrome coronavirus-2 (SARS-CoV-2), an enveloped RNA virus(2). The number of confirmed COVID-19 cases is growing rapidly. There have been nearly 45 million confirmed cases globally and over 1 million deaths at the time of this manuscript submission, according to the data of the World Health Organization (https://covid19.who.int/).

COVID-19 could cause a wide variety of symptoms such as dyspnea, headache, myalgia, fever, cough, and altered smell or taste(3). The most of morbidity and mortality from this disease have been originated pulmonary(4). This disease also maybe sometimes asymptomatic. Besides, many researches are supported that the illness can also present with non-respiratory symptoms such as abdominal pain, and diarrhea $(5,6)$.

The presence of these nonspecific symptoms may cause delaying the diagnosis of the disease. The early diagnosis plays an important role to prevent the spread of the disease and avoids the rapidly deteriorating patients with COVID-19(7). The standard method for a confirmed diagnosis of the disease is a real-time polymerase chain reaction (RT-PCR). There are some shortcomings related to this diagnostic test (RT-PCR; 
typically, nasopharyngeal swab). These shortcomings include false-positive results, false-negative results, and late diagnosis(8). Chest computed tomography (CT) is not recommended as the first diagnostic tool, but it can be used as an important complementary diagnostic tool in addition to RT-PCR(7). The most common imaging findings on chest $\mathrm{CT}$ are ground-glass opacities without lung cavitation, discrete pulmonary nodules, and bilateral patchy shadowing. Also, can be seen some patterns such as bronchial wall thickening, linear opacities(1).

However, diagnosis in asymptomatic patients or patients with non-respiratory symptoms remains a big concern. Many patients with COVID-19 pneumonia can admit to the clinic with symptoms such as abdominal pain and renal colic. These abnormal symptoms can have resulted in requests for abdominal CT. In this study, we aimed to evaluate the incidence of missed diagnosed COVID-19 pneumonia on abdominal CT performed in patients admitted to our urology outpatient clinic.

\section{Study Design}

We reviewed the files of patients who were admitted to the urology outpatient clinic from 1 April to 1 November. We selected this date as an origin. Because, in our country, it was announced COVID-19 was spread all over Turkey on 1 April (https://tr.wikipedia.org/wiki/T\%C3\%BCrkiye\%27de_COVID-19_pandemisi).

We included the patients who performed abdominal CT at the urology outpatient clinic for any reason. We recorded demographic data of these patients such as age, sex, complaints of admission, and abdominal CT findings. Also, we examined the lung bases findings on abdominal CT imaging of these patients.

The lung bases findings on these abdominal CT were accepted with COVID-19 pneumonia and were classified as incidental (Figure1, Figure 2);

- Ground-glass opacities

- Bilateral patchy shadowing

- Bronchial wall thickening

- Linear opacities

- Discrete pulmonary nodules $(7,9)$.

We excluded patients with pulmonary symptoms and previously diagnosed with COVID-19. Also, patients without abdominal CT were excluded.

We evaluated the rates of missed diagnosed COVID-19 pneumonia detection on the lung base images of abdominal CT. The pulmonary findings on the abdominal CT associated with COVID-19 are reviewed with the lung window settings owing to findings is not visible on abdominal window settings.

These CT imaging, each of the patients, were evaluated by three authors (MSO, MBH, and BY) to increase the reliability of the analysis. These authors have been actively involved in the diagnosis and treatment of COVID-19 since the outbreak began. Therefore, they are sufficiently familiar with lung findings in the diagnosis of this disease. The evaluated CT findings were confirmed by the author EE who is a specialist Infectious Diseases and Clinical Microbiology.

Each study was reviewed with the lung, abdominal, and mediastinal window settings. All CT studies were performed by high-resolution thin section datasets (Slice thickness of $2 \mathrm{~mm}$ and an increment of $1 \mathrm{~mm}$ were used).

The institutional human research ethics committee approved the protocol $20 / 483$. The analysis and data collection were performed after written informed consent was obtained from all patients according to Helsinki Declaration.

\section{Statistical Analysis}


All statistical analyses were performed using Statistical Package for Social Sciences volume 22.0 (IBM Corp.; IL, Chicago, USA). Non-parametric tests were used for parameters that have been shown difference from the normal distribution. The parameters with normal distribution was analyzed by parametric tests. The variables with continuous normally distributed were expressed as mean \pm SD. The categorical variables were presented as percentage and the variables without normally distributed were expressed as median and interquartile ranges (IQRs).

\section{Results}

In this present study, the files of 1081 patients were reviewed. 57 of these patients were excluded from the study due to their CT did not contain any pulmonary images. After the criteria were applied 1024 patients were included in this study. The mean age of patients $44.3 \pm 14.9$ years (3-101 years). 357 (34.9\%) of the patients were female, and $667(65.1 \%)$ were male.

We observed that $99(9.7 \%)$ of these patients had findings related to missed diagnosed COVID-19 pneumonia on the lung base images of abdominal CT. Although 885 (86.4\%) of patients had no pathological pulmonary findings, 40 (3.9) patients had other pathological pulmonary findings (non-related COVID-19) (such as atelectasis, pulmonary fibrosis, pleural effusion, etc.) (Figure 3).

The mean age of patients who had findings COVID-19 were $56.4 \pm 14.7$ years (22-92 years). 35 (35.4\%) of these 99 patients were female, $64(64.6 \%)$ were male; these findings were similar to the patients without abnormal pulmonary imaging (Chi-Square; p: 0.930). Also, there were no abnormal urinary system findings on abdominal CT imaging 39 of these 99 patients. These patients had only pulmonary findings related to COVID-19 (Figure 4).

We observed that 688 (67.2\%) of these patients had abnormal urinary system findings (such as ureteral stones, renal stones, renal cell carcinoma, simple renal cyst, etc.). There were 288 patients with ureteral stones, 261 patients with renal calculi, 44 patients with simple renal cyst, 8 patients with angiomyolipoma, and 86 patients with other urinary tract pathologies (renal malignancies, hydronephrosis, ureteropelvic junction obstruction, etc.).

\section{Discussion}

The symptoms of COVID-19 vary widely ranged across. The most of infected patients are asymptomatic. It can lead to only self-limiting respiratory illness in symptomatic patients. The most common medical conditions requiring hospitalization are related to diseases of pulmonary such as pneumonia, hypoxia. But in some patients, it could cause severe multisystem disease that can be fatal $(1,3,4)$. So, the quickly and correctly diagnosis of the disease is a substantial entity. RT-PCR is the standard diagnostic method(10). However, the use of these tools could have impractical for clinical management due to related to concerns such as delaying the diagnosis or false-negative results. So, it may be necessary to use supportive diagnostic methods such as laboratory findings or imaging methods(10).

The chest radiographs commonly have no diagnostic value in the early stages of the disease. Thus, they are not useful clinically for diagnosis. But, pulmonary CT may include many findings even before symptom onset(9). Even if routine CT imaging is not recommended for diagnosis of COVID-19 pneumonia(11), for its contributions to the diagnosis, the rapid advice guideline recommended the pulmonary CT as strongly for patients with suspected COVID-19(12). The patients who are not yet suspected COVID-19 may have been characteristic pulmonary findings of COVID-19 pneumonia. Also, CT findings are could be support COVID-19 in patients who are investigated to novel viral pneumonia infection(13). The commonly characteristic CT patterns for COVID are included in; ground-glass opacities, bilateral or multilobar involvement, cavitation, bilateral patchy shadowing, septal or pleural thickening, linear opacities, and discrete pulmonary nodules etc. $(14,15)$. The most of cases with confirmed COVID-19 have initial abnormal CT manifestations. Even, some of these patients have not any symptoms related to COVID-19 and were imaged only for screening(16). Similarly, none of the patients in our study have any pulmonary symptoms. These patients were asymptomatic, or have non-pulmonary symptoms. 
The radiologic modalities in urology practice have been used widespread in recent years(17). This widespread use of these tools such as ultrasonography, or abdominal CT enables incidental detection and early diagnosis of diseases such as renal cell carcinoma(18). Although there were some exceptions, most abdominal CT studies include a consistent portion, especially lung bases, of pulmonary component(19). In the study by $\mathrm{Vu}$ et al, it was presented that three patients without respiratory symptoms had doubtful imaging findings for COVID-19. None of these patients had pulmonary CT. Abnormal imaging findings were seen on the pulmonary component of abdominal CT in two of these patients(20). Similarly, other studies examining this issue consisted of case series(21-24). Moreover, in their study, Barkmeier et al. stated that there were 42 patients with COVID-19 have abdominal CT that visualized lung bases. They also stated that $79 \%$ of these patients have abnormal CT findings on lung bases(13). In this present study, we found that $9.7 \%$ of the patients had abnormal CT findings consistent with COVID-19 pneumonia. These findings were supported by another study which was showing the screening of the lung base images may benefit the diagnosis of COVID-19 for patients with abdominal CT(25).

COVID-19 has no directly pathological effect on the urinary tract. But, this disease, which takes hold all over the world, affects significantly daily clinical practice(26). In this pandemic time, many hospitals have served only patients with COVID-19, and the hospitals' workload has increased considerably. This statement has led to an increase in the requirement for healthcare(27). So, a pragmatic approach must be necessary for the management of this disease. All medical doctors including urologists should increase their awareness of this disease. They are playing an important role in the fight against COVID-19 during this pandemic. They also must be aware of the potential signs of illness(28). Based on these findings, we evaluated the lung base findings of the patients' abdominal CT. We found that a significant part of the patients has typical findings of COVID-19 pneumonia. This rate is a portion of the patients we encounter in our daily outpatient clinic, which cannot be ignored. So, it is too important to the assessment of imaging hallmarks for effective patient treatment and management with increasing concerns about the SARS-CoV-2 outbreak.

This present study has some limitations. First, we evaluated the patients who were admitted only to the urology outpatient clinic. So, the study population maybe not enough for generalization. Second, we could not correlate all of these patients with RT-PCR. Despite these limitations, this present study has significant findings of COVID-19 management.

\section{Conclusions}

COVID-19 disease has become a pandemic all over the world. All healthcare professionals including urologists play an active role in the diagnosis and treatment of this disease. So, it should be kept in mind that COVID19 pneumonia should be evaluated in patients admitted to the urology outpatient clinic with renal colic or abdominal pain.,

\section{Abbreviations}

COVID-19: The novel coronavirus disease 2019

CT: Computed tomography

RT-PCR: Real-time polymerase chain reaction

SARS-CoV-2: Severe acute respiratory syndrome coronavirus-2

\section{Declarations}

\section{Conflicts of interests}

The authors declare that they have no competing interests exist.

\section{Ethics declarations}

The institutional human research ethics committee approved the protocol 20/483 (Health Science University, Hamidiye Scientific Research Ethics Committee). The analysis and data collection were performed following 
the Declaration of Helsinki after written informed consent was obtained from all patients.

\section{Funding}

The authors declare that they have no financial competing interests exist.

\section{Availability of data and material}

Data are available on request.

\section{Code availability}

Not applicable

\section{Acknowledgments}

Not applicaple

\section{References}

1. Guan W-j, Ni Z-y, Hu Y, Liang W-h, Ou C-q, He J-x, et al. Clinical characteristics of coronavirus disease 2019 in China. New England journal of medicine. 2020;382(18):1708-20.

2. Zhu N, Zhang D, Wang W, Li X, Yang B, Song J, et al. A novel coronavirus from patients with pneumonia in China, 2019. New England Journal of Medicine. 2020.

3. Yang W, Cao Q, Qin L, Wang X, Cheng Z, Pan A, et al. Clinical characteristics and imaging manifestations of the 2019 novel coronavirus disease (COVID-19): A multi-center study in Wenzhou city, Zhejiang, China. Journal of Infection. 2020.

4. Wu Z, McGoogan JM. Characteristics of and important lessons from the coronavirus disease 2019 (COVID19) outbreak in China: summary of a report of 72314 cases from the Chinese Center for Disease Control and Prevention. Jama. 2020;323(13):1239-42.

5. Price-Haywood EG, Burton J, Fort D, Seoane L. Hospitalization and mortality among black patients and white patients with Covid-19. New England Journal of Medicine. 2020.

6. Huang C, Wang Y, Li X, Ren L, Zhao J, Hu Y, et al. Clinical features of patients infected with 2019 novel coronavirus in Wuhan, China. The lancet. 2020;395(10223):497-506.

7. Singh D, Kumar V, Kaur M. Classification of COVID-19 patients from chest CT images using multiobjective differential evolution-based convolutional neural networks. European Journal of Clinical Microbiology \& Infectious Diseases. 2020:1-11.

8. Woloshin S, Patel N, Kesselheim AS. False Negative Tests for SARS-CoV-2 Infection-Challenges and Implications. New England Journal of Medicine. 2020.

9. Pan Y, Guan H, Zhou S, Wang Y, Li Q, Zhu T, et al. Initial CT findings and temporal changes in patients with the novel coronavirus pneumonia (2019-nCoV): a study of 63 patients in Wuhan, China. European radiology. 2020:1-4.

10. Cohen PA, Hall L, John JN, Rapoport AB, editors. The early natural history of SARS-CoV-2 infection: Clinical observations from an urban, ambulatory COVID-19 clinic. Mayo Clinic Proceedings; 2020: Elsevier.

11. Simpson S, Kay FU, Abbara S, Bhalla S, Chung JH, Chung M, et al. Radiological Society of North America Expert Consensus Statement on Reporting Chest CT Findings Related to COVID-19. Endorsed by the Society of Thoracic Radiology, the American College of Radiology, and RSNA. Radiology: Cardiothoracic Imaging. 2020;2(2):e200152.

12. Jin Y-H, Cai L, Cheng Z-S, Cheng H, Deng T, Fan Y-P, et al. A rapid advice guideline for the diagnosis and treatment of 2019 novel coronavirus (2019-nCoV) infected pneumonia (standard version). Military Medical Research. 2020;7(1):4. 
13. Barkmeier DT, Stein EB, Bojicic K, Otemuyiwa B, Vummidi D, Chughtai A, et al. Abdominal CT in COVID-19 patients: incidence, indications, and findings. Abdominal Radiology. 2020:1-7.

14. Fang Y, Zhang H, Xu Y, Xie J, Pang P, Ji W. CT manifestations of two cases of 2019 novel coronavirus (2019-nCoV) pneumonia. Radiology. 2020;295(1):208-9.

15. Lei J, Li J, Li X, Qi X. CT imaging of the 2019 novel coronavirus (2019-nCoV) pneumonia. Radiology. 2020;295(1):18-.

16. Salehi S, Abedi A, Balakrishnan S, Gholamrezanezhad A. Coronavirus disease 2019 (COVID-19): a systematic review of imaging findings in 919 patients. American Journal of Roentgenology. 2020:1-7.

17. Ficarra V, Prayer-Galetti T, Novella G, Bratti E, Maffei N, Dal Bianco M, et al. Incidental detection beyond pathological factors as prognostic predictor of renal cell carcinoma. European urology. 2003;43(6):6639.

18. Tsui K-H, Shvarts O, Smith RB, FIGLIN R, de KERNION JB, BELLDEGRUN A. Renal cell carcinoma: prognostic significance of incidentally detected tumors. The Journal of urology. 2000;163(2):426-30.

19. Rinaldi MF, Bartalena T, Giannelli G, Rinaldi G, Sverzellati N, Canini R, et al. Incidental lung nodules on CT examinations of the abdomen: prevalence and reporting rates in the PACS era. European journal of radiology. 2010;74(3):e84-e8.

20. Vu D, Ruggiero M, Choi WS, Masri D, Flyer M, Shyknevsky I, et al. Three unsuspected CT diagnoses of COVID-19. Emergency radiology. 2020:1-4.

21. Abolyazid S, Alshareef S, Abdullah N, Khalil A, Hamza N, Salem A. COVID-19 pneumonia identified by $\mathrm{CT}$ of the abdomen: A report of three emergency patients presenting with abdominal pain. Radiology Case Reports. 2020;15(11):2090-4.

22. Sendi AA, Saggat DF, Alzahrani SJ. Incidental typical COVID-19 appearance on the lung bases, visualized at abdominal CT for a patient that presented with abdominal pain and nausea. Radiology case reports. 2020;15(8):1238-41.

23. Siegel A, Chang PJ, Jarou ZJ, Paushter DM, Harmath CB, Arevalo JB, et al. Lung base findings of coronavirus disease (COVID-19) on abdominal CT in patients with predominant gastrointestinal symptoms. American Journal of Roentgenology. 2020:1-3.

24. Voutsinas N, Toussie D, Jacobi A, Bernheim A, Chung M. Incidental CT findings in the lungs in COVID19 patients presenting with abdominal pain. Clinical Imaging. 2020.

25. Hamilton N, Adam G, Ifan D, Lam S, Johnson K, Vedwan K, et al. Diagnostic utility of additional whole-chest $\mathrm{CT}$ as part of an acute abdominal pain CT imaging pathway during the COVID-19 pandemic. Clinical Radiology. 2020.

26. Gul M, Kaynar M, Yildiz M, Batur AF, Akand M, Kilic O, et al. The Increased Risk of Complicated Ureteral Stones in the Era of COVID-19 Pandemic. Journal of Endourology. 2020;34(8):882-6.

27. Bozkurt O, Sen V, Irer B, Sagnak L, Onal B, Tanidir Y, et al. Nation-wide analysis of the impact of Covid19 pandemic on daily urology practice in Turkey. International Journal of Clinical Practice. 2020:e13735.

28. Wallis CJ, Catto JW, Finelli A, Glaser AW, Gore JL, Loeb S, et al. The Impact of the COVID-19 Pandemic on Genitourinary Cancer Care: Re-envisioning the Future. European urology. 2020.

\section{Figure Legends}

Figure 1: COVID-19 pneumonia findings on abdominal CT

Figure 2: COVID-19 pneumonia findings on abdominal CT

Figure 3: The pulmonary findings of lung base on abdominal CT 
Figure 4: Urinary system findings of patients with and without COVID-19
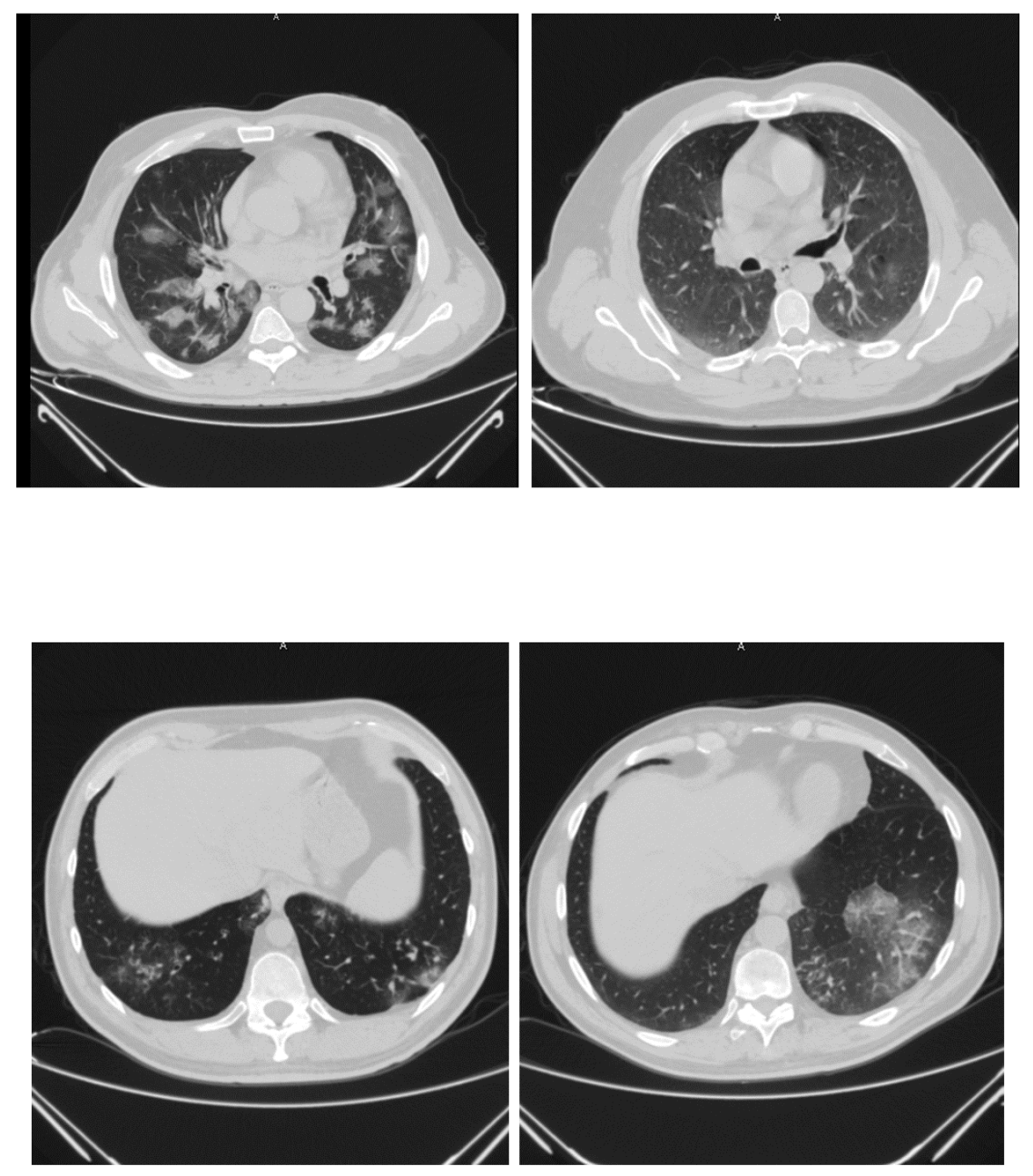


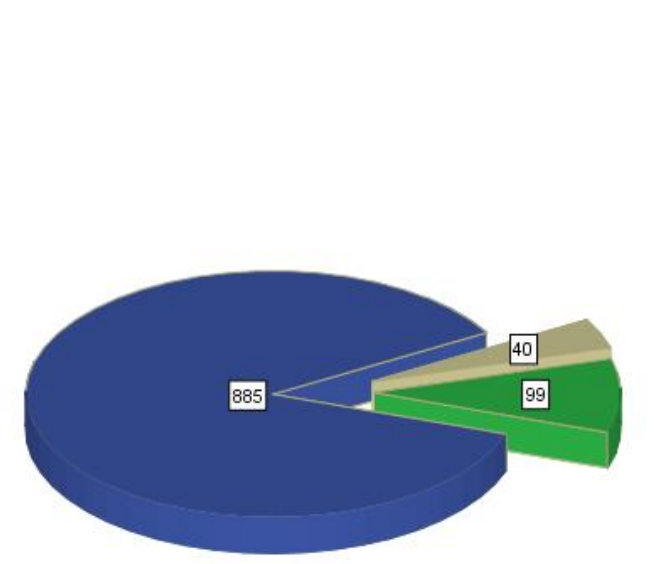
No pathological findings
COVID-19

Other pulmonary findings

\section{Hosted file}

Figure 4.pdf available at https://authorea.com/users/357674/articles/519740-incidence-ofmissed-diagnosed-covid-19-pneumonia-on-patients-who-admitted-urology-outpatient-clinic 